\title{
Budgets Balancing Algorithms for the Projects Assignment
}

\author{
Mahdi Jemmali \\ Department of Computer Science and Information \\ College of Science in Zulfi, Majmaah University \\ Majmaah, 11952, Saudi Arabia
}

\begin{abstract}
This paper focused on the resolution of the project's assignment problem. Several heuristics have been developed and proposed in this paper to serve as lower bounds to our studied problem. In a developing country, it is interesting to make an equitable distribution of projects in different cities in order to guarantee equality and regional development. Each project is characterized by its budget. The problem is to find an appropriate schedule to assign all projects to all cities. This appropriate schedule seeking the maximization of the budget in the city that having the minimum budget. In this paper, six heuristics were proposed to carry out the objective of resolving the studied problem. The experimental results show that the algorithm given by the heuristic $P_{6}^{r}$ outperforms all other heuristics cited in this paper.
\end{abstract} ment

Keywords-Heuristic; scheduling algorithms; project assign-

\section{INTRODUCTION}

The regional development is a very important thing for each country. Appropriate distribution of resources is primordial to guarantee social and economic stability. The economic cycle may directly be impacted by the development of cities. The principal main of this research is the allocation of the project. The problem is based on the fact that we have several regions and several projects to distribute to the regions. The principal goal of this work is to maximize the budget for the city that having the minimum budget. This goal will be presented as a maximization of the minimum total budget problem. This problem can be solved approximately using several heuristics. Given a set of projects to be distributed to different cities (regions). Each project has its budget and will be entirely assigned to a chosen city. Seeking the balancing of the budgets to all regions, we apply some heuristics to schedule projects on the regions to maximize the minimal total budget [1]. In our work, it is assuming that all regions are identical and have the same economic characteristic, this is meaning all regions can attract all given projects. Heuristics will be used to give a feasible dispatching solution of all projects. The unequal distribution of income across regions is studied in [2] which authors presented in the research work the theory of optimal control.

Other researchers use the operations research field applied in the allocation of investment using the dynamic programming problems with linear form. The regional allocation problem was studied to found the optimal solution proprieties [3].

In our paper we propose to make dispatching of projects on cities by using several heuristics of the follows problem.
Minimizing the maximum total completion time for parallel machine $P \| C_{\text {min }}$ as defined in [1]. For the latter problem, an optimal method using a branch-and-bound algorithm was given in [1]. The developed algorithm used essentially the proposed tight lower and upper bounds.

In [4], the authors developed a method for multi-mode resource with cost availability problems in the project assignment. Other approach is proposed based on differential search $(D S)$ related to the conditions and constraints of the resource project assignment problem [5]. Recently, the author in [6] proposed three approximate Solutions to solve the problem that treated the project revenues but no experimental result is given in the latter work. Some randomized variants were developed as heuristics to solve approximately the problem of the aircraft turbines engines in [7]. Indeed, authors in the latter work show that the solution of the problem can be given by solving the maximization of the minimum completion time. This problem is the same one applied in the studied case in this paper. On other work type, authors in [8], reveal the problem of allocation resources by resolving the problem using a nearoptimal solution. Recently, an equity algorithm is applied on routers to ensure the balancing assignment of data through different routers [9]. This latter research work utilizes the same approach of our studied problem. This presented paper is organized as follows. In Section 2, we present the studied problem and we give some details for the problem in general. Section 3, presents six developed lower bounds through several heuristics for the studied problem. The experimental study is done in Section 4.

\section{PROBlem Description}

The problem proposed in this paper is the maximization of the minimum total budget. This problem is described as follows. Let a set $P s$ that determined by the $n_{p}$ projects. These projects must be distributed to a fixed number of regions $n_{r}$. We index each region $R_{i}$ by the index $i$. The estimated budget of each project $j$ is denoted by $b_{j}$. We can't assign the same project to different regions. Each project will be assign at only one region at a time. The studied problem concerns the maximization of the minimum total budget given by $R_{\min }$. The cumulative budget of the project $j$ is given by the variable $C b_{j}$.

Example 1: Assume that the number of projects is $5\left(n_{p}=\right.$ 5 ) and the number of regions is $2\left(n_{r}=2\right)$. The budget $b_{j}$ for each project $j$ is depicted in Table I. 
TABLE I. BUDGET-PROJECTS VALUES.

\begin{tabular}{cccccc}
\hline$j$ & 1 & 2 & 3 & 4 & 5 \\
\hline$b_{j}$ & 7 & 2 & 9 & 3 & 12 \\
\hline
\end{tabular}

We schedule all projects on the regions applying a given algorithm. The schedule gave a result which is depicted in Fig. 1. From the latter figure, we see that region 1 has projects 3 , 1 and 4 . However, for region 2, projects 5 and 2 are assigned.

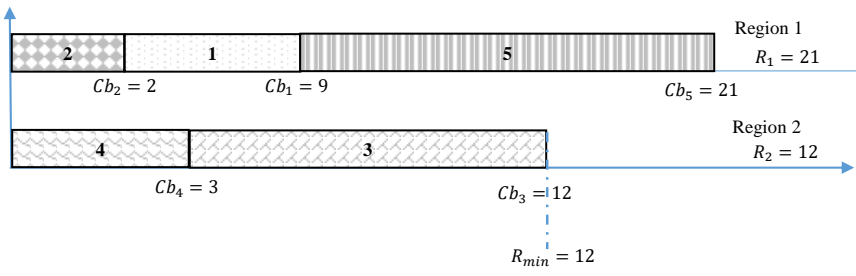

Fig. 1. Project-regions assignment.

From Fig. 1, we see that the region 1 has a total budget of 21 . Nonetheless, region 2 has a total budget of 12 . For this example, the maximum assigned budget is 21 . However, the minimum total assigned budget is $R_{\min }=12$. The goal is to give a permutation (assignment) that maximizes the minimum of the assigned total budget of $R_{\text {min }}$. So, we have to search for other more performed heuristics that offers a minimum total budget better than 12 .

Using the three-field notation in [10], the studied problem can be denoted as $P \| C_{\text {min }}$.

\section{LOWER BOUNDS}

In this research work, we give a comparison of the performance of the proposed lower bounds for the studied problem with the well-known dispatching rule $L P T$ given in [1]. Indeed, we develop six lower bounds. Two strategies are proposed in this section to develop the six algorithms. The first strategy is based on the probabilistic method applied to the choice of the project which will be assigned to the regions. The second strategy is based on the Multi-Fit algorithm to be used for the proposed problem.

\section{A. Non-increasing Budgets Order Heuristic (NIB)}

In this dispatching rule, all projects will be ordered in the non-increasing order of its budgets. After that, we assign the project having the greatest budget on the region that has the minimum total budget at this time.

\section{B. Probabilistic based Heuristics $\left(P_{k}^{r}\right)$}

For this type of heuristic, our study is essentially based on the selection of the project having the biggest budget to be assigned to the region that has the minimum total budget with a probabilistic approach. The probabilistic selection between the $k$ biggest project with $k \in\{2,3, \cdots, 6\}$ for the heuristics $\left\{P_{2}^{r}, \cdots, P_{6}^{r}\right\}$, respectively.

The chosen project is selected among the $k$ first project having the biggest budget with probability $\alpha$. We denote by
$R D(a, b)$ the randomly function that return an integer between two limits $(a, b)$. The calculation of the probabilistic selection is fixed as follows:

- $\quad r=R D(1, k)$. The picked project will be the $r^{t h}$ biggest non-assigned project. We assign the chosen project to the region that have a minimum total budget.

- Denoted by $U_{p}$ the number of non-assigned projects. If $U_{p}<k$, then $r=R D\left(1, U_{p}\right)$.

For a fixed integer $k$, we choose the iteration number as limit $=1500$. The heuristic of the probabilistic project-region is the following:

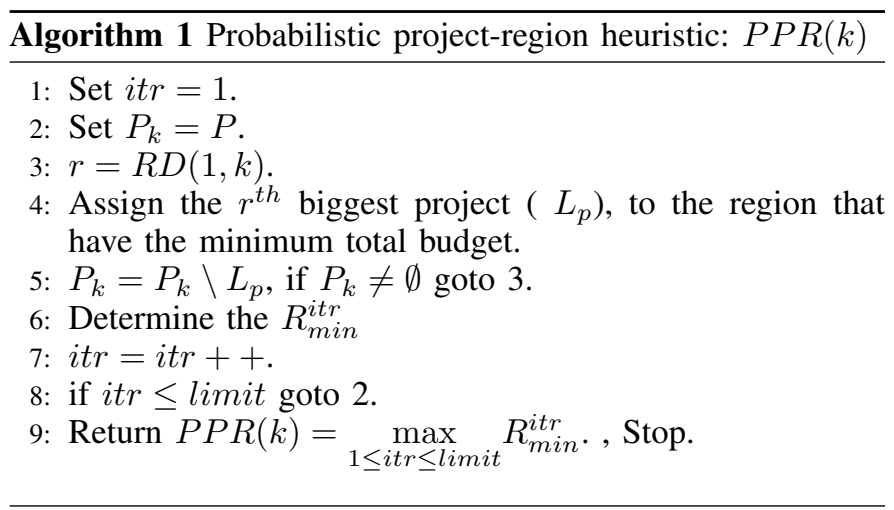

The above algorithm is given for a fixed $k$.

The Algorithm 1 is used when the $k$ value is fixed in advance. To develop the algorithm of the proposed heuristic we must iterate the above as the number between 2 and $k$. Indeed, the final algorithm which represents the probabilistic based heuristics $P_{k}^{r}$ needs the iteration of $P P R(j)$ from 2 to $k$. The calculation of the value of the heuristic $P_{k}^{r}$ for a predetermined $k$ is elaborated by as following algorithm.

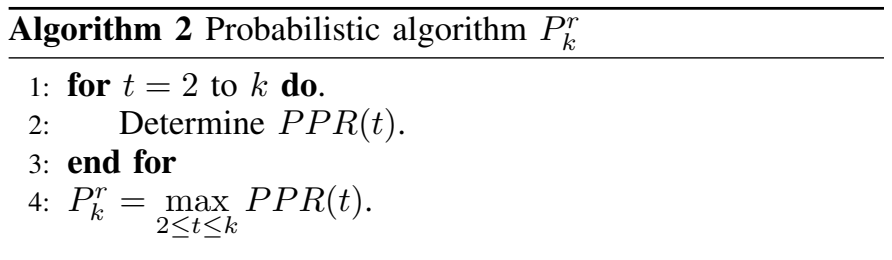

\section{Multi-fit Projects based Heuristic (MFP)}

The Multi-Fit heuristic is developed in several works in literature. For our proposed research work we propose an adaptive Multi-Fit heuristic. The main idea is based to found the minimum total budget when all projects will be assigned to the given regions.

The heuristic is based on the utilization of the bin-packing approach. For our case study, the region is considered as bin and the project as an item. Once the bin capacity is fixed, the First Fit Decreasing $(F F D)$ algorithm is used to fit projects to the bin. The first step of the algorithm is to order projects according to their budget such that $b_{1} \geq b_{2} \geq \ldots b_{n_{p}}$.

Let $L=\max \left(b_{1}, b_{n_{r}}+b_{n_{r}+1},\left\lceil\frac{\sum_{p=1}^{n_{p}} b_{p}}{n_{r}}\right\rceil\right)$ and $U$ is the value given by applying the LPT heuristic for the problem 
$P \| C_{\max }$. The number of reputation ite is fixed in advance. This number is used for the iteration of FFD. We set ite= 135 and $n_{b}$ denoted the number of bins used when we apply the FFD routine.

The algorithm below describe all instructions to calculate $M F P$.

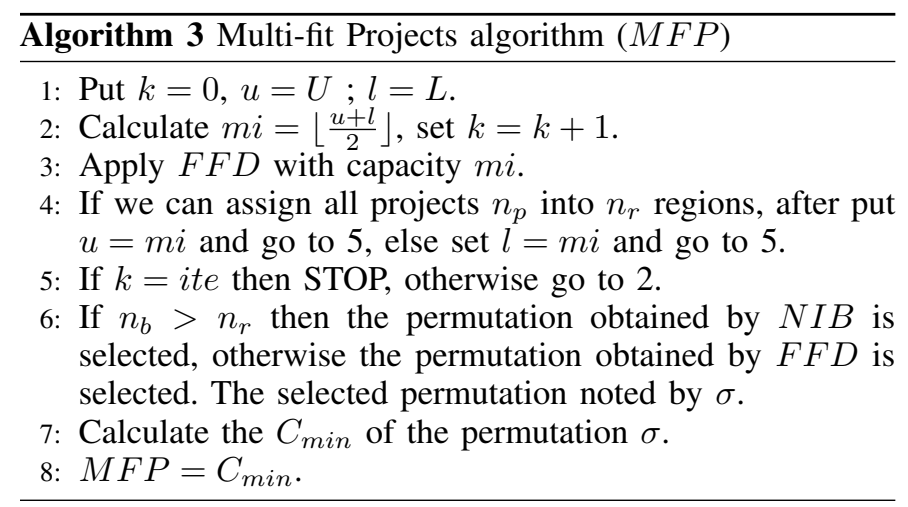

Example 2: Let $n_{p}=10$ and $n_{r}=2$. The following table represent the budget of each project.

TABLE II. INSTANCE OF BUDGETS-PROJECTS FOR HEURISTIC $M F P$

\begin{tabular}{ccccccccccc}
\hline$j$ & 1 & 2 & 3 & 4 & 5 & 6 & 7 & 8 & 9 & 10 \\
\hline$b_{j}$ & 81 & 30 & 33 & 66 & 53 & 62 & 66 & 59 & 96 & 55 \\
\hline
\end{tabular}

Applying the NIB heuristic for the instances given in Table II, the result will as follows. For the first region, we assign the projects $\{2,4,5,8,9\}$ with a total budget of $U=304$. For the second region, we assign the projects $\{1,3,6,7,10\}$ with total budget $L=297$. Then, $m i=\left\lfloor\frac{304+297}{2}\right\rfloor=302$. Applying FFD function with capacity 302 and the teen items which is corresponding to projects in Table II. The region 1 has the projects $\{1,4,8,9\}$ and the second region will have the projects $\{2,3,5,6,7,10\}$. The first region has a total budget of 302 however the second one has a total budget of 299 . Thus, $C_{\min }=299$. On the other hand, the value returned by the heuristic $L P T(N I B)$ is 297 . So, the result obtained by $M F P$ is better than NIB.

\section{EXPERIMENTAL RESULTS}

We adopt several instances of classes to discuss with results and show the developed algorithms comparing with the $L P T$ cited in previous research works.

All algorithms proposed in this paper are coded with $\mathrm{Mi}$ crosoft Visual C++ (Version 2013). The developed algorithms were coded and running on an Intel(R) Core(TM) i5-3337U CPU@1.8 GHz. The operating system is windows 10.

The developed algorithms were running on five types of sets of instances. These types of instances were generated as presented in [1]. We generate the budget $b_{j}$ using to different distributions. Each distribution represents a class. The distribution of the classes are as following:

- $\quad$ Class $1: b_{j}$ is in $U[30,100]$.

- Class 2: $b_{j}$ is in $U[50,300]$.
- Class 3: $b_{j}$ is in $U[200,500]$.

- Class 4: $b_{j}$ is in $N[50,150]$.

- Class $5: b_{j}$ is in $N[25,500]$.

with $U[a, b]$ represent the uniform distribution between $[a, b]$ and $N[a, b]$ represent the normal distribution. The generated instances was obtained by the choice of $n_{p}, n_{r}$ and Class. The pair $\left(n_{p}, n_{r}\right)$ can have several affectation values. Indeed, we choose the affectation values given in Table III below.

TABLE III. THE $n_{p}$ AND $n_{r}$ DISTRIBUTION

\begin{tabular}{cc}
\hline$n_{p}$ & $n_{r}$ \\
\hline 10 & $2,3,5$ \\
25,50 & $2,3,5,10,15$ \\
100,250 & $3,5,10,15,25,30$ \\
300,500 & $10,15,30,50$ \\
\hline
\end{tabular}

Table III shows that we have in total of 1650 instances. Several indicators are given in this paper to show the performance of the proposed algorithms compared with the LPT values proposed in the previous work and presented in [1]. The indicators are given as follows.

- $\quad L m$ the best heuristic value returned after running of algorithms.

- $\quad L$ the discussed lower bound.

- $G A P=\frac{L m-L}{L} \times 100$.

- Time the running time execution for heuristic. This time will be in seconds.

- Perc the percentage among the (1650) instances that the condition $L m=L$ is satisfied.

Table IV present the overall percentage and average time for all proposed algorithms.

TABLE IV. OVERALL PERCENTAGE AND AVERAGE TIME COMPARISON

\begin{tabular}{cccccccc} 
& $L P T$ & $M F P$ & $P_{2}^{r}$ & $P_{3}^{r}$ & $P_{4}^{r}$ & $P_{5}^{r}$ & $P_{6}^{r}$ \\
\hline Perc & $16.2 \%$ & $16.1 \%$ & $41.6 \%$ & $52.2 \%$ & $58.5 \%$ & $68.2 \%$ & $84.3 \%$ \\
\hline Time & 0.000 & 0.023 & 0.047 & 0.095 & 0.139 & 0.192 & 0.237 \\
\hline
\end{tabular}

The above table shows that the heuristic that conducts the best value is $P_{6}^{r}$ with $\operatorname{Perc}=84.3 \%$ and Time $=0.237 \mathrm{~s}$, compared to $L P T$ rule that the corresponding percentage is $16.2 \%$.

The following table represents the behavior of Gap according to $n_{p}$.

TABLE V. VARIATION OF $G A P$ ACCORDING TO $n_{p}$

\begin{tabular}{cccccccc}
\hline$n_{p}$ & $L P T$ & $M F P$ & $P_{2}^{r}$ & $P_{3}^{r}$ & $P_{4}^{r}$ & $P_{5}^{r}$ & $P_{6}^{r}$ \\
\hline 10 & 1.67 & 2.17 & 0.47 & 0.08 & 0.03 & 0.00 & 0.00 \\
25 & 1.78 & 4.60 & 0.77 & 0.46 & 0.33 & 0.18 & 0.08 \\
50 & 0.84 & 7.19 & 0.24 & 0.11 & 0.07 & 0.04 & 0.03 \\
100 & 0.66 & 30.24 & 0.38 & 0.27 & 0.18 & 0.10 & 0.05 \\
250 & 0.15 & 5.12 & 0.10 & 0.07 & 0.05 & 0.03 & 0.02 \\
300 & 0.22 & 1.88 & 0.12 & 0.08 & 0.05 & 0.03 & 0.01 \\
500 & 0.08 & 2.35 & 0.05 & 0.04 & 0.03 & 0.02 & 0.01 \\
\hline
\end{tabular}

Table $\mathrm{V}$ shows that when varying the number of projects $\left(n_{p}\right)$, the performance of the developed algorithms varies. The 
above table shows that there aren't any depends on the number of projects and the calculated $G A P$. For heuristics [ $L P T$, $M F P, P_{2}^{r}, P_{3}^{r}, P_{4}^{r}, P_{5}^{r}$ and $\left.P_{6}^{r}\right]$ the best $G A P$ value was obtained for the following values of $n_{p}[500,300,500,500$, $(10,500), 10,10]$, respectively. Also this table shows that algorithm $P_{6}^{r}$ having the best $G A P$ when $n_{p}=10$ and the rule $L P T$ having the worst $G A P$ when $n_{p}=500$.

The execution time for heuristics in Table $\mathrm{V}$ is given in Table VI.

TABLE VI. Time FOR EACH HEURISTIC AND EACH CORRESPONDING $n_{p}$

\begin{tabular}{cccccccc}
\hline$n_{p}$ & $L P T$ & $M F P$ & $P_{2}^{r}$ & $P_{3}^{r}$ & $P_{4}^{r}$ & $P_{5}^{r}$ & $P_{6}^{r}$ \\
\hline 10 & 0.000 & 0.002 & 0.001 & 0.002 & 0.004 & 0.005 & 0.004 \\
25 & 0.000 & 0.004 & 0.003 & 0.007 & 0.009 & 0.012 & 0.015 \\
50 & 0.000 & 0.006 & 0.006 & 0.012 & 0.017 & 0.032 & 0.027 \\
100 & 0.000 & 0.010 & 0.017 & 0.034 & 0.052 & 0.070 & 0.095 \\
250 & 0.000 & 0.025 & 0.058 & 0.120 & 0.173 & 0.230 & 0.291 \\
300 & 0.000 & 0.060 & 0.086 & 0.171 & 0.255 & 0.346 & 0.437 \\
500 & 0.000 & 0.062 & 0.174 & 0.354 & 0.522 & 0.727 & 0.886 \\
\hline
\end{tabular}

Table VII display the results of the $G A P$ value according to the number of regions $n_{r}$. The worst $G A P$ value is given for algorithm $M F P$ when $n_{r}=30$ and the best $G A P$ value is given for algorithm $P_{6}^{r}$ when $n_{r}=3$ or $n_{r}=50$.

TABLE VII. VARIATION OF THE $G A P$ VALUES ACCORDING TO $n_{r}$

\begin{tabular}{cccccccc}
\hline$n_{r}$ & $L P T$ & $M F P$ & $P_{2}^{r}$ & $P_{3}^{r}$ & $P_{4}^{r}$ & $P_{5}^{r}$ & $P_{6}^{r}$ \\
\hline 2 & 1.01 & 0.40 & 0.20 & 0.14 & 0.11 & 0.09 & 0.08 \\
3 & 1.52 & 1.28 & 0.59 & 0.19 & 0.09 & 0.04 & 0.01 \\
5 & 0.39 & 0.90 & 0.05 & 0.03 & 0.03 & 0.02 & 0.02 \\
10 & 0.97 & 3.29 & 0.51 & 0.30 & 0.21 & 0.11 & 0.03 \\
15 & 0.42 & 7.22 & 0.25 & 0.20 & 0.15 & 0.10 & 0.05 \\
25 & 0.80 & 2.70 & 0.41 & 0.25 & 0.15 & 0.07 & 0.03 \\
30 & 0.28 & 51.14 & 0.15 & 0.09 & 0.05 & 0.03 & 0.02 \\
50 & 0.27 & 4.72 & 0.16 & 0.12 & 0.07 & 0.04 & 0.01 \\
\hline
\end{tabular}

The execution time for heuristics in Table VII is given in Table VIII.

TABLE VIII. Time FOR EACH HEURISTIC AND EACH CORRESPONDING $n_{r}$

\begin{tabular}{cccccccc}
\hline$n_{r}$ & $L P T$ & $M F P$ & $P_{2}^{r}$ & $P_{3}^{r}$ & $P_{4}^{r}$ & $P_{5}^{r}$ & $P_{6}^{r}$ \\
\hline 2 & 0.000 & 0.003 & 0.004 & 0.005 & 0.007 & 0.013 & 0.013 \\
3 & 0.000 & 0.001 & 0.013 & 0.025 & 0.037 & 0.054 & 0.070 \\
5 & 0.000 & 0.016 & 0.014 & 0.029 & 0.041 & 0.054 & 0.067 \\
10 & 0.000 & 0.040 & 0.050 & 0.101 & 0.149 & 0.202 & 0.251 \\
15 & 0.000 & 0.014 & 0.055 & 0.109 & 0.163 & 0.223 & 0.278 \\
25 & 0.000 & 0.033 & 0.045 & 0.093 & 0.135 & 0.183 & 0.230 \\
30 & 0.000 & 0.022 & 0.092 & 0.186 & 0.279 & 0.400 & 0.474 \\
50 & 0.000 & 0.088 & 0.154 & 0.322 & 0.465 & 0.619 & 0.790 \\
\hline
\end{tabular}

The Table IX present the variation of the calculated $G A P$ according to Class. It is clear to see that the class instances and the $G A P$ values are not correlated.

TABLE IX. VARIATION OF THE $G A P$ VALUES ACCORDING TO Class

\begin{tabular}{cccccccc}
\hline Class & LPT & MFP & $P_{2}^{r}$ & $P_{3}^{r}$ & $P_{4}^{r}$ & $P_{5}^{r}$ & $P_{6}^{r}$ \\
\hline 1 & 0.69 & 8.60 & 0.26 & 0.13 & 0.09 & 0.04 & 0.02 \\
2 & 0.70 & 5.71 & 0.22 & 0.11 & 0.07 & 0.04 & 0.02 \\
3 & 0.72 & 9.88 & 0.34 & 0.17 & 0.11 & 0.06 & 0.03 \\
4 & 1.17 & 16.23 & 0.45 & 0.24 & 0.16 & 0.08 & 0.02 \\
5 & 0.38 & 4.21 & 0.26 & 0.19 & 0.15 & 0.10 & 0.07 \\
\hline
\end{tabular}

The execution time for heuristics and Table IX is given in Table X.

TABLE X. Time FOR EACH HEURISTIC AND EACH CORRESPONDING Class

\begin{tabular}{cccccccc}
\hline Class & LPT & MFP & $P_{2}^{r}$ & $P_{3}^{r}$ & $P_{4}^{r}$ & $P_{5}^{r}$ & $P_{6}^{r}$ \\
\hline 1 & 0.000 & 0.027 & 0.046 & 0.091 & 0.136 & 0.190 & 0.236 \\
2 & 0.000 & 0.021 & 0.048 & 0.094 & 0.140 & 0.205 & 0.242 \\
3 & 0.000 & 0.028 & 0.047 & 0.098 & 0.140 & 0.192 & 0.238 \\
4 & 0.000 & 0.003 & 0.047 & 0.096 & 0.141 & 0.188 & 0.234 \\
5 & 0.000 & 0.033 & 0.046 & 0.093 & 0.140 & 0.186 & 0.237 \\
\hline
\end{tabular}

For more statistics about the gap values, we present Table XI. From the Table XI, we can see that the maximum $G A P$ is 171.59 obtained when $n_{p}=100$ and $n_{r}=30$ for the heuristic $M F P$.

TABLE XI. DETAILS FOR ALL ALGORITHMS

\begin{tabular}{|c|c|c|c|c|c|c|c|c|}
\hline$n_{p}$ & $n_{r}$ & $L P T$ & $M F P$ & $P_{2}^{r}$ & $P_{3}^{r}$ & $P_{4}^{r}$ & $P_{5}^{r}$ & $P_{6}^{r}$ \\
\hline \multirow{3}{*}{10} & 2 & 1.08 & 0.59 & 0.03 & 0.00 & 0.00 & 0.00 & 0.00 \\
\hline & 3 & 3.92 & 3.14 & 1.39 & 0.24 & 0.08 & 0.01 & 0.00 \\
\hline & 5 & 0.03 & 2.79 & 0.00 & 0.00 & 0.00 & 0.00 & 0.00 \\
\hline \multirow{5}{*}{25} & 2 & 1.91 & 0.57 & 0.56 & 0.41 & 0.34 & 0.27 & 0.24 \\
\hline & 3 & 1.64 & 1.94 & 0.73 & 0.26 & 0.11 & 0.04 & 0.00 \\
\hline & 5 & 1.30 & 1.11 & 0.13 & 0.08 & 0.11 & 0.07 & 0.06 \\
\hline & 10 & 4.05 & 16.87 & 2.46 & 1.53 & 1.07 & 0.54 & 0.10 \\
\hline & 15 & 0.00 & 2.52 & 0.00 & 0.00 & 0.00 & 0.00 & 0.00 \\
\hline \multirow{5}{*}{50} & 2 & 0.06 & 0.03 & 0.00 & 0.00 & 0.00 & 0.00 & 0.00 \\
\hline & 3 & 1.57 & 0.73 & 0.49 & 0.21 & 0.10 & 0.05 & 0.01 \\
\hline & 5 & 0.41 & 0.39 & 0.06 & 0.02 & 0.02 & 0.02 & 0.03 \\
\hline & 10 & 1.04 & 1.81 & 0.28 & 0.12 & 0.08 & 0.08 & 0.06 \\
\hline & 15 & 1.11 & 32.99 & 0.40 & 0.20 & 0.14 & 0.07 & 0.07 \\
\hline \multirow{6}{*}{100} & 3 & 0.34 & 0.42 & 0.26 & 0.16 & 0.08 & 0.04 & 0.02 \\
\hline & 5 & 0.16 & 0.18 & 0.06 & 0.03 & 0.02 & 0.01 & 0.01 \\
\hline & 10 & 0.48 & 0.54 & 0.20 & 0.11 & 0.08 & 0.05 & 0.02 \\
\hline & 15 & 1.05 & 4.10 & 0.83 & 0.78 & 0.62 & 0.39 & 0.16 \\
\hline & 25 & 1.27 & 4.62 & 0.62 & 0.38 & 0.23 & 0.11 & 0.04 \\
\hline & 30 & 0.68 & 171.59 & 0.32 & 0.16 & 0.07 & 0.03 & 0.03 \\
\hline \multirow{6}{*}{250} & 3 & 0.11 & 0.17 & 0.10 & 0.08 & 0.05 & 0.03 & 0.01 \\
\hline & 5 & 0.04 & 0.04 & 0.01 & 0.01 & 0.00 & 0.00 & 0.00 \\
\hline & 10 & 0.12 & 0.23 & 0.05 & 0.02 & 0.01 & 0.01 & 0.00 \\
\hline & 15 & 0.21 & 1.47 & 0.17 & 0.16 & 0.13 & 0.09 & 0.06 \\
\hline & 25 & 0.32 & 0.78 & 0.20 & 0.12 & 0.08 & 0.04 & 0.01 \\
\hline & 30 & 0.10 & 28.02 & 0.05 & 0.03 & 0.03 & 0.01 & 0.00 \\
\hline \multirow{4}{*}{300} & 10 & 0.09 & 0.17 & 0.04 & 0.02 & 0.01 & 0.01 & 0.00 \\
\hline & 15 & 0.14 & 0.22 & 0.08 & 0.05 & 0.04 & 0.03 & 0.00 \\
\hline & 30 & 0.28 & 1.69 & 0.18 & 0.11 & 0.08 & 0.03 & 0.01 \\
\hline & 50 & 0.36 & 5.43 & 0.20 & 0.15 & 0.08 & 0.05 & 0.02 \\
\hline \multirow{4}{*}{500} & 10 & 0.05 & 0.10 & 0.03 & 0.02 & 0.01 & 0.01 & 0.00 \\
\hline & 15 & 0.02 & 2.00 & 0.01 & 0.00 & 0.00 & 0.00 & 0.00 \\
\hline & 30 & 0.06 & 3.27 & 0.05 & 0.05 & 0.04 & 0.04 & 0.04 \\
\hline & 50 & 0.19 & 4.02 & 0.13 & 0.09 & 0.06 & 0.03 & 0.01 \\
\hline
\end{tabular}

\section{Conclusion}

This paper treats the problem of the distribution of different projects having several budgets. The problem is NP-hard. We proposed in this paper several heuristics to give approximates solutions for the studied problem. The algorithms given in this work are tested through some classes of instances generated uniformly based on classes already proposed in the literature review. The results show that the probabilistic and iterative method outperforms the Multi-fit and dispatching rule algorithms. The results show that the algorithm $P_{6}^{r}$ has the best $G A P$ value through the tested instances. Future work can be 
done regarding the performance of other heuristics and the exact solution of the studied problem.

\section{ACKNOWLEDGMENT}

The authors would like to thank the Deanship of Scientific Research at Majmaah University for supporting this work under Project Number No. R-1441-30.

\section{REFERENCES}

[1] M. Haouari and M. Jemmali, "Maximizing the minimum completion time on parallel machines," 4OR, vol. 6, no. 4, pp. 375-392, 2008.

[2] S. Alexiadis, "Regional allocation of investment: Implications for regional policy," Procedia Economics and Finance, vol. 9, pp. 176-180, 2014.

[3] Y. Ohtsuki, "Regional allocation of public investment in an n-region economy," Journal of Regional Science, vol. 11, no. 2, pp. 225-233, 1971.

[4] J.-J. Qi, Y.-J. Liu, H.-T. Lei, and B. Guo, "Solving the multi-mode resource availability cost problem in project scheduling based on modified particle swarm optimization," Arabian Journal for Science and Engineering, vol. 39, no. 6, pp. 5279-5288, 2014.
[5] N. Rahmani, V. Zeighami, and R. Akbari, "A study on the performance of differential search algorithm for single mode resource constrained project scheduling problem," Decision Science Letters, vol. 4, no. 4, pp. 537-550, 2015.

[6] M. Jemmali, "Approximate solutions for the projects revenues assignment problem," Communications in Mathematics and Applications, vol. 10 , no. 3,2019

[7] M. Jemmali, L. K. B. Melhim, and M. Alharbi, "Randomized-variants lower bounds for gas turbines aircraft engines," in World Congress on Global Optimization. Springer, 2019, pp. 949-956.

[8] N. R. Devanur, K. Jain, B. Sivan, and C. A. Wilkens, "Near optimal online algorithms and fast approximation algorithms for resource allocation problems," Journal of the ACM (JACM), vol. 66, no. 1, p. 7, 2019.

[9] M. Jemmali and H. Alquhayz, "Equity data distribution algorithms on identical routers," in International Conference on Innovative Computing and Communications. Springer, 2020, pp. 297-305.

[10] E. L. Lawler, J. K. Lenstra, A. H. R. Kan, and D. B. Shmoys, "Sequencing and scheduling: Algorithms and complexity," Handbooks in operations research and management science, vol. 4, pp. 445-522, 1993. 